\title{
A Note on PIN Polynomials and PRIN Rational Functions
}

\author{
Michael Z. Q. Chen
}

\begin{abstract}
This brief presents a necessary and sufficient condition for testing positive, real, imaginary, and negative rational functions. A related term, the positive, imaginary, and negative polynomial, is defined and two necessary and sufficient conditions for testing it are given.
\end{abstract}

Index Terms-Hurwitz polynomials, positive, imaginary, and negative (PIN) polynomials, positive, real, imaginary, and negative (PRIN ) property, PRIN rational functions.

\section{INTRODUCTION}

$\mathbf{T}$ He main subject under study in this work is the positive, real, imaginary, and negative (PRIN) property and PRIN rational functions. The term PRIN was coined in [10] and it is adopted here for brevity. It is a well-known way of defining reactance functions (Foster's Reactance Theorem [4]) in passive network synthesis, where a special class of positive-real functions can be realized using reactive elements only (capacitors, inductors and possibly transformers) [1], [6]. The PRIN property was also used in [10] to prove Kharitonov's Theorem which is a stability theorem for a class of polynomials with each coefficient varying independently in a specified (but arbitrary) interval [7], [8]. The PRIN property also has a potential link with the design of positive real functions which are of interest in the study of absolute stability and hyper-stability [3], [5], [9]. However, the PRIN property has been wrongly associated with Hurwitz polynomials in [6], [10], which motivated this brief. This brief has a potential impact in both circuit synthesis theory and system stability theory.

This brief is organized as follows. Section II reviews the positive, imaginary and negative (PIN) polynomials, the PRIN property and the PRIN functions and presents some existing results in corrected forms. Conclusion is given in Section III.

\section{PRIN PROPERTY AND PRIN FUNCTIONS}

In this section, we will review the PRIN property and PRIN rational functions. However, in the sequence of logic, we first define an important property of polynomials, the PIN property, which will lead us to the PRIN property. Its form has appeared in [6].

Definition 1: A polynomial $f(s)$ has the PIN (positive, imaginary and negative) property if:

$$
\begin{array}{lll}
|f(s)|>|f(-s)|, & \text { for } & \operatorname{Re}(s)>0 \\
|f(s)|=|f(-s)|, & \text { for } & \operatorname{Re}(s)=0 \\
|f(s)|<|f(-s)|, & \text { for } & \operatorname{Re}(s)<0 .
\end{array}
$$

Manuscript received on June 20, 2007; revised October 10, 2007. This paper was recommended by Associate Editor A. Vicino.

The author is with the Department of Engineering, University of Leicester, Leicester LE1 7RH, U.K.(e-mail: mc274@1e.ac.uk/mzqchen@gmail.com).

Digital Object Identifier 10.1109/TCSII.2007.912686
In [6], it was stated that a polynomial has the PIN property if and only if it is Hurwitz [zeroes in the open left half-plane (OLHP)], which is not entirely correct. This incorrect statement subsequently leads to the incorrect results on the PRIN property in [6], which was used again in [1] and [10]. Now a correct statement is presented in Proposition 2 and a proof is given. Note that Proposition 2 is a necessary and sufficient condition for testing PIN polynomials and it is the basis of all the results subsequently derived.

Proposition 2: A polynomial $f(s)$ has the PIN property if and only if $f(s)$ has all its roots in the closed left half-plane (CLHP) but not all on the imaginary axis.

Proof: (Only if.)

Suppose to the contrary, $f(s)$ has all roots on the imaginary axis or has root(s) in the open right half-plane (ORHP). If $f(s)$ has all roots on the imaginary axis, then $|f(s)|=|f(-s)|$ for all $s$, which is a contradiction. If $f(s)$ has a root, say $s_{0}$, in the ORHP, then $\left|f\left(s_{0}\right)\right|=0$ while $\left|f\left(-s_{0}\right)\right| \geq 0$, contradicting the condition that $|f(s)|>|f(-s)|$ for $\operatorname{Re}(s)>0$.

(If.)

Write

$$
\begin{aligned}
f(s)=\left(s+r_{1}\right) \ldots\left(s+r_{n}\right)\left[\left(s+\sigma_{1}\right)^{2}+\omega_{1}^{2}\right] & \\
& \ldots\left[\left(s+\sigma_{m}\right)^{2}+\omega_{m}^{2}\right]\left(s^{2}+\omega_{m+1}^{2}\right) \ldots\left(s^{2}+\omega_{m+k}^{2}\right)
\end{aligned}
$$

where $r_{i}>0(i=1, \ldots, n)$ are roots on the negative real axis, $\sigma_{i} \pm j \omega_{i}\left(\sigma_{i}>0\right.$ and $\left.i=1, \ldots, m\right)$ are complex conjugate roots in the OLHP and $\pm j \omega_{m+i}(i=1, \ldots, k)$ are the roots on the imaginary axis.

For any $s_{0}=r_{0}+j \omega_{0}$ with $r_{0}>0$ and $\omega_{0} \geq 0$, we can see that $\left|\left(r_{0}+j \omega_{0}\right)^{2}+\omega_{m+i}^{2}\right|=\left|\left(-r_{0}-j \omega_{0}\right)^{2}+\omega_{m+i}^{2}\right|$ with $i=1, \ldots, k,\left|\left(\sigma_{i}+r_{0}+j \omega_{0}\right)^{2}+\omega_{i}^{2}\right|>\left|\left(\sigma_{i}-r_{0}-j \omega_{0}\right)^{2}+\omega_{i}^{2}\right|$ with $i=1, \ldots, m$ and $\left|r_{i}+r_{0}+j \omega_{0}\right|>\left|r_{i}-r_{0}-j \omega_{0}\right|$ with $i=1, \ldots, n$. Since we have at least one real root or one pair of complex roots in the OLHP, $|f(s)|>|f(-s)|$ for $\operatorname{Re}(s)>0$.

Similarly, we can prove that $|f(s)|=|f(-s)|$ for $\operatorname{Re}(s)=0$ and $|f(s)|<|f(-s)|$ for $\operatorname{Re}(s)<0$.

In view of Proposition 2, results derived from the PIN property in the literature - in particular the PRIN property, have to be modified as well. The remainder of this section is devoted to some results derived from Proposition 2 that have appeared in the literature in incorrect forms, which might have or will lead to other incorrect results.

We now define PRIN rational functions [6], [10].

Definition 3: A rational function $\psi(s)$ has the PRIN property if

$$
\begin{array}{llll}
\operatorname{Re}(\psi)>0, & \text { for } & \operatorname{Re}(s)>0 \\
\operatorname{Re}(\psi)=0, & \text { for } & \operatorname{Re}(s)=0 \\
\operatorname{Re}(\psi)<0, & \text { for } & \operatorname{Re}(s)<0 .
\end{array}
$$


Proposition 4 is a necessary and sufficient condition for testing PRIN rational functions. A similar but incorrect form of Proposition 4 from [6] has appeared in [10], which was used to prove a major result [10, Theorem 2.2] and had previously been used to prove [10, Theorem 2.1] in [2]. Proposition 5 is in fact another necessary and sufficient condition for testing PIN polynomials and its proof makes use of the PRIN property as defined in (2). The proofs of Propositions 4 and 5 are not essentially different from those presented in [6] and are therefore omitted.

Proposition 4: Consider a polynomial $f(s)=m(s)+n(s)$ with $m(s)$ and $n(s)$ being the even and odd parts respectively. The ratio of even and odd parts $\psi(s)=m(s) / n(s)$ is a PRIN rational function if and only if $f(s)$ has all its roots in the CLHP but not all on the imaginary axis. ${ }^{1}$

Proof: See [6] for details.

Proposition 5: Consider a polynomial $f(s)=m(s)+n(s)$ with $m(s)$ and $n(s)$ being the even and odd parts, respectively. Then $f(s)$ has all its roots in the CLHP but not all on the imaginary axis if and only if the ratio of even and odd parts $\psi(s)=$ $m(s) / n(s)$ has simple poles on the imaginary axis only, and with positive real residues at these poles.

Proof: See [6] for details.

The following theorem is a direct consequence of of Proposition 4. A similar but incorrect form [10, Theorem 2.2] has appeared in [10]-[12], where it was a major result leading to the alternative proof of the stability theorem by Kharitonov [7], [8].

Theorem 6: Consider a PIN polynomial $f_{1}(s)=m(s)+$ $n(s)$, where $m(s)$ and $n(s)$ being the even and odd parts, respectively. Then the polynomial $f_{2}(s)=k_{1} m(s)+k_{2} n(s)$ is also a PIN polynomial for all $k_{1}>0$ and $k_{2}>0$.

Proof: It is immediate from Proposition 4. For any given $\psi(s)=m(s) / n(s)$ that has the property $(2),\left[k_{1} m(s)\right] /\left[k_{2} n(s)\right]$ also has the property (2). The result follows.

\section{CONCLUSION}

The PRIN property has been associated with Hurwitz polynomials in the literature. However, it was shown in this brief that it

${ }^{1}$ If all the roots of $f(s)$ are on the imaginary axis, then the odd part $n(s) \equiv 0$. is actually related to polynomials with their roots in the CLHP but not all on the imaginary axis. Two necessary and sufficient conditions for testing such polynomials were presented. In turn, a necessary and sufficient condition for testing PRIN rational functions was given. Some existing results were presented in corrected forms.

\section{ACKNOWLEDGMENT}

Thanks are due to Dr. H.-T. Zhang (University of Cambridge, Cambridge, U.K.) and anonymous reviewers for their help in improving the presentation and correcting the misprints of the manuscript.

\section{REFERENCES}

[1] N. Balabanian, Network Synthesis. Upper Saddle River, NJ: PrenticeHall, 1958.

[2] N. K. Bose, "A system theoretic approach to stability of set of polynomials," Contemp. Math., vol. AMS-47, pp. 25-34, 1985.

[3] M. de la Sen, "A method for general design of positive real functions," IEEE Trans. Circuits Syst. I, Fundam. Theory Appl., vol. 45, no. 7, pp. 764-769, Jul. 1998.

[4] R. M. Foster, "A reactance theorem," Bell Syst. Tech. J., vol. 3, pp. 259-267, 1924.

[5] J. Gregor, "On the design of positive real functions," IEEE Trans. Circuits Syst. I, Fundam. Theory Appl., vol. 43, pp. 945-947, Nov. 1996.

[6] E. A. Guillemin, The Mathematics of Circuit Analysis. Cambridge, MA: M.I.T. Press, 1949.

[7] V. L. Kharitonov, "Asymptotic stability of an equilibrium position of a family of systems of linear differential equations," Differentsial'nye Uravneniya, vol. 14, pp. 2086-2088, 1978.

[8] V. L. Kharitonov, "Asymptotic stability of an equilibrium position of a family of systems of linear differential equations," Differential Equations, vol. 14, pp. 1483-1485, 1979.

[9] H. J. Marquez and C. J. Damaren, "On the design of strictly positive real transfer functions," IEEE Trans. Circuits Syst. I, Fundam. Theory Appl., vol. 42, no. 4, pp. 214-218, Apr. 1995.

[10] V. V. Patel and K. B. Datta, "Classification of units in $H_{\infty}$ and an alternative proof Kharitonov's theorem," IEEE Trans. Circuits Syst. I, Fundam. Theory Appl., vol. 44, no. 5, pp. 454-458, May 1997.

[11] V. V. Patel and K. B. Datta, "Comments on on Hurwitz stable polynomials and strictly positive real transfer functions," IEEE Trans. Circuits Syst. I, Fundam. Theory Appl., vol. 48, no. 1, pp. 128-129, Jan. 2001.

[12] L. Wang and W. Yu, "On Hurwitz stable polynomials and strictly positive real transfer functions," IEEE Trans. Circuits Syst. I, Fundam. Theory Appl., vol. 48, no. 1, pp. 127-128, Jan. 2001. 